\title{
Circuit Design of a Wide Tuning Range CMOS VCO with Automatic Amplitude Control
}

\author{
Jiwei Chen \\ Institute of Microelectronics \\ Tsinghua University \\ Beijing 100084, P. R. China \\ chjw98@mails.tsinghua.edu.cn
}

\author{
Bingxue Shi \\ Institute of Microelectronics \\ Tsinghua University \\ Beijing 100084, P. R. China \\ shibx@tsinghua.edu.cn
}

\begin{abstract}
A novel tuning mechanism to greatly widen the frequency tuning range of VCO is presented. A tuning range of over $50 \%$ has been achieved for a CMOS VCO with the adoption of this technique. Moreover, a constant amplitude control loop is also incorporated in this circuit, ensuring stable oscillation amplitude over the whole tuning range.
\end{abstract}

\section{Categories and Subject Descriptors}

B.7.1 [Integrated Circuits]: Types and Design Styles - VLSI (very large scale integration).

\section{General Terms Design}

Keywords VCO, CMOS, tuning range, automatic amplitude control.

\section{INTRODUCTION}

As a very important block for RF transceiver system, voltage controlled oscillator impacts many performances of the whole system. With the ceaseless advance of CMOS technology, it is becoming more and more attractive for the integration and implementation of such a system.

The traditional LC oscillator can be modeled with a RLC parallel network, in which a variable capacitor is used for frequency tuning [1]. But due to the absence of good varactor compatible with CMOS process, the tuning range of integrated CMOS LCtype VCO is typically lower than $20 \%$ of the centre frequency [2, $3,4]$.

Since the lot-to-lot process variations in on-chip capacitance and inductance may be as large as $20 \%$, the oscillation frequency may also have an error of $20 \%$ or more for lot-to-lot difference. The tuning range of VCO must be wide enough to compensate for the fluctuation.

A CMOS ring oscillator with $50 \%$ tuning range has been demonstrated in Ref. [5]. A LC oscillator which is tunable from $1.34 \mathrm{GHz}$ to $2.14 \mathrm{GHz}$ is reported in Ref. [6], by connecting two inductors in parallel, and changing the inductance by switching one of them from cut-off to turn-on. It needs more area-hungered inductors, and the tuning range is limited by the ratio of the two inductors in parallel.

Permission to make digital or hard copies of all or part of this work for personal or classroom use is granted without fee provided that copies are not made or distributed for profit or commercial advantage and that copies bear this notice and the full citation on the first page. To copy otherwise, or republish, to post on servers or to redistribute to lists, requires prior specific permission and/or a fee.

GLSVLSI'03, April 28-29, 2003, Washington, DC, USA.

COPYRIGHT 2003 ACM 1-58113-677-3/03/0004 ..\$5.00
Here a novel tuning mechanism to broaden the frequency tuning range of LC VCO is proposed. By tuning the resistance in series with a capacitor, a tuning range of over $50 \%$ has been achieved for a CMOS VCO with the adoption of this technique. Moreover, a constant amplitude control loop is also incorporated in this circuit, ensuring stable oscillation amplitude over the whole tuning range.

\section{TUNING PRINCIPLE}

As shown in Figure 1, the circuit in (a) containing serially connected resistor $R_{S}$ and capacitor $C_{S}$ can be transformed to its parallel form, showing in (b) of this figure.

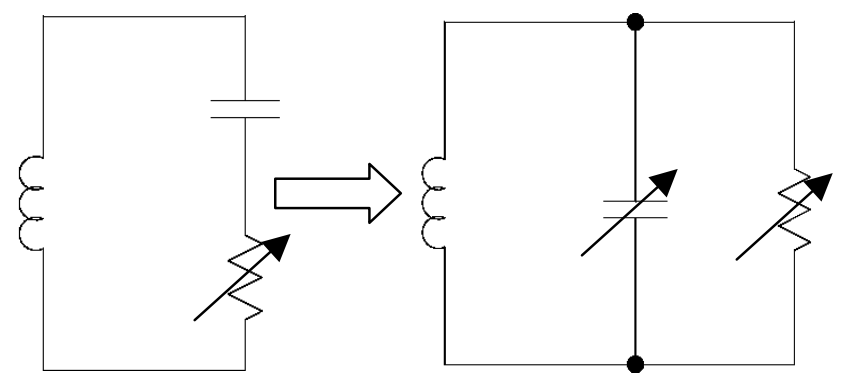

Equating the real parts and imaginary parts of the impedances of the series and parallel $R C$ sections respectively, the following transformation formulas can be achieved:

$$
\begin{aligned}
& C_{P}=\frac{C_{S}}{1+\omega^{2} C_{S}^{2} R_{S}^{2}} \\
& R_{P}=R_{S}+\frac{1}{\omega^{2} C_{S}^{2} R_{S}}
\end{aligned}
$$

It can be seen from equation (1) that $C_{P}$ is variable if $R_{S}$ is a tunable resistor controlled by a voltage. The oscillation frequency of this LC is given by:

$$
f_{0}=\frac{1}{2 \pi \sqrt{L C_{P}}}
$$


By tuning the resistance of $R_{S}$, the equivalent parallel capacitance $C_{P}$ is changed, and then the oscillation frequency is changed also. This is the tuning principle of this circuit.

The tunable resistor $R_{S}$ can be realized through a MOSFET with its gate connected to a control voltage, which can be implemented very easily with CMOS technology. The capacitor $C_{S}$ can be realized with MIM (metal-insulatormetal) capacitor, which is also a standard process in mixedsignal CMOS process. Thus this novel approach eliminates the need of varactors.

It can be seen from equation (1) that when $R_{S}$ approaches zero, $C_{P}$ will be close to $C_{S}$, and when $R_{S}$ approaches infinite, $C_{P}$ will be close zero. So theoretically, the frequency tuning range can be:

$$
\left(\frac{1}{2 \pi \sqrt{L C_{S}}}, \infty\right)
$$

For on-chip MIM capacitor, there is a parasitic capacitor between the lower plate of it and the substrate. The value of this parasitic capacitor can be one tenth or more of the nominal value of the MIM capacitor generally, and it determines a rough upper limit of the frequency range. If we nominate this capacitance as $C_{o x}$, the frequency tuning range can be revised as

$$
\left(\frac{1}{2 \pi \sqrt{L C_{S}}}, \frac{1}{2 \pi \sqrt{L C_{o x}}}\right)
$$

In above equation, the ratio of the upper limit to the lower limit is about three.

But in reality, the tune-on resistance of a MOSFET can not be zero, and there exists parasitic capacitance of on-chip MOSFETtype resistor. MIM capacitor and other devices connected to this point will set an upper limit to the oscillation frequency much lower than infinite.

\section{PROPOSED CIRCUIT ANALYSIS}

The differential VCO is shown in Figure 2. The on-chip spiral inductor $L$ and MIM capacitor $C$ forms the resonant tank, and MOSFET M0 is controlled by the tuning voltage as the variable resistor. The cross coupled differential pair formed by M1 and M2 is used to keep the oscillation.

The frequency sensitivity is an important parameter of VCO, determining by the characteristic of M0. The turn-on resistance of M0 may change sharply when the control voltage is near its threshold voltage, and a small variation of the control voltage may cause a large fluctuation of oscillation frequency. To alleviate this problem, several types of MOS transistors with different threshold voltages are connected in parallel.

As shown in equation (2), the equivalent parallel resistance $R_{P}$ will decreases to a minimum value then increases when M0 changes from cut-off to fully turn-on. This minimum value is given by:

$$
\min R_{P}=\frac{2}{\omega C_{S}}
$$

At this time, the network is lossiest. So the bias point and size of M1 and M2 must be determined to have a minimum acceptable transconductance for guaranteeing oscillation. This is expressed as:

$\min g_{m}=\frac{1}{\min R_{P}}=\frac{\omega C_{S}}{2}$

Since the equivalent parallel resistance $R_{P}$ may varies vastly during the whole tuning range, the oscillation amplitude can also be very different. Too large oscillation amplitude may destroy the gate of MOSFET, and this problem is more and more serious as the gate oxide becomes thinner and thinner with improve of CMOS technology. Not only that, too large oscillation amplitude can also degrade the phase noise performance [4]. Furthermore, stable oscillation amplitude over the whole frequency tuning range is desirable for mixers. These make the amplitude regulation necessary [5-6].

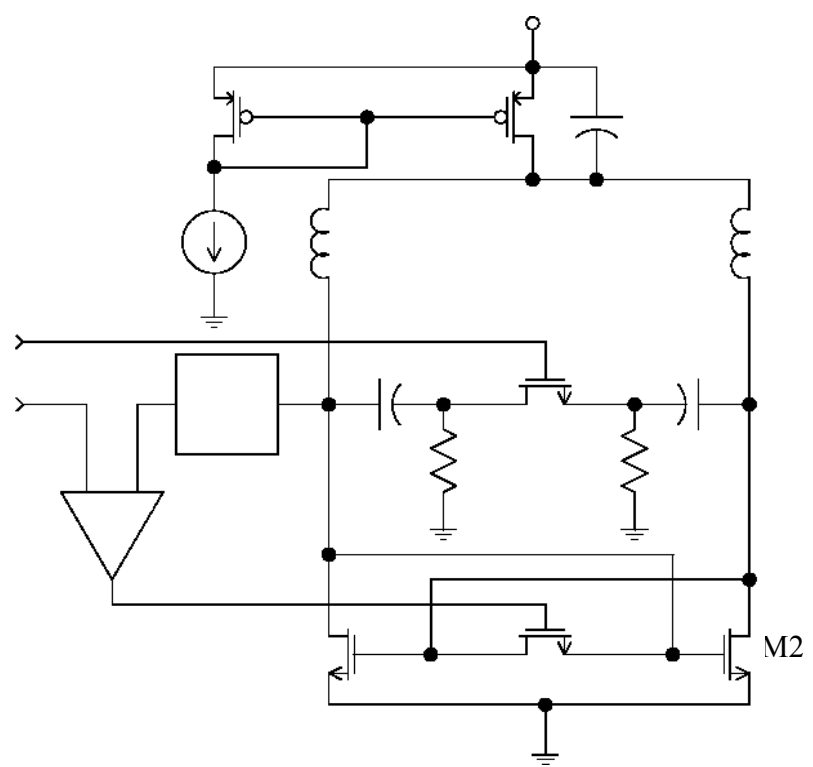

Figure 2. Schematic of the proposed VCO

Figure 3 shows the schematic detail of the peak detector. It output is controlled by two currents: push and pull. When the input terminal is larger than the output, a large current is injected to the output terminal, making its voltage rise fast; when the input terminal is smaller than the output, a small current is drained from the output terminal, making its voltage decrease slowly. Thus the output can track the peak value of the input oscillation. The values of the push current and pull current decide the attack time and the decay time of this circuit, which should be determined by applications.

The schematic detail of the OTA is shown in Figure 4. Its pull current $I_{P U L L}$ is a constant current, while its push current $I_{P U S H}$ is decided by the difference of the input terminal voltage and the reference voltage $V_{R E F}$. When $V_{I N}$ equals $V_{R E F}$, then $I_{P U S H}$ equals $I_{P U L L}$, and the voltage of the output terminal keeps stable. The NMOS transistor MC is connected as a capacitor, saving silicon area and achieving faster charge time due to its low capacitance when $V_{\text {OUT }}$ is smaller than its threshold voltage, compared with traditional MIM capacitor. 


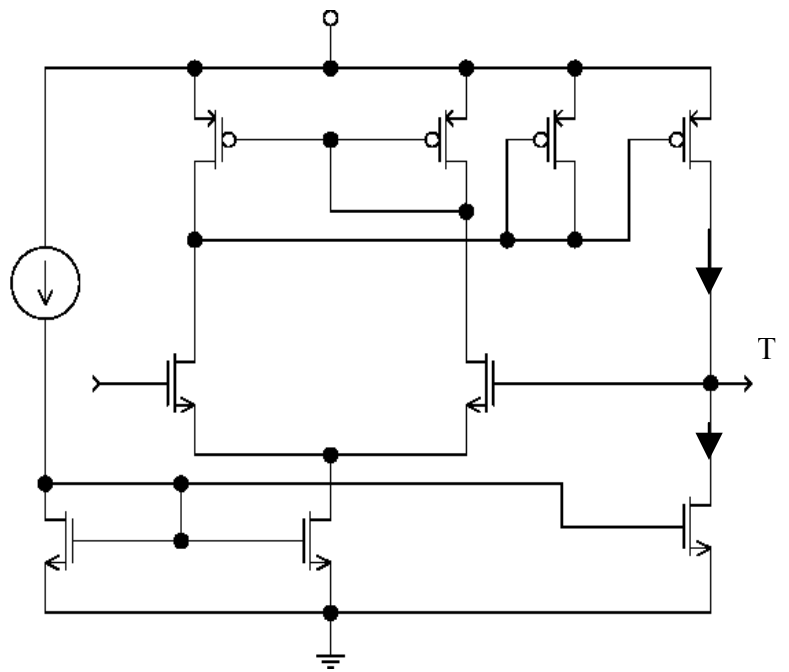

Figure 3. Schematic of the peak detector

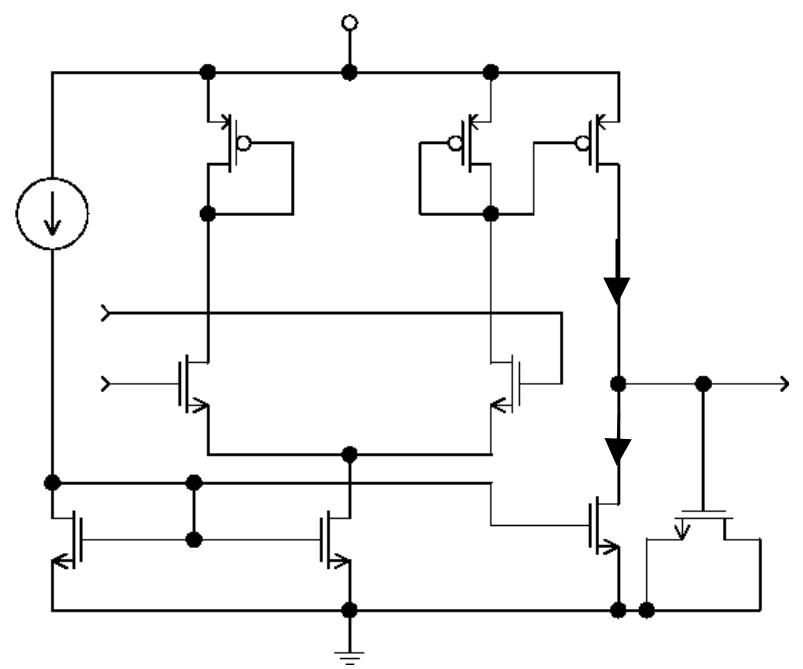

Figure 4: Schematic of the OTA

\section{SIMULATION RESULTS}

This circuit is designed and simulated by using $0.18 \mu \mathrm{m}$ mixedsignal CMOS process with $1.8-\mathrm{V}$ supply voltage to the correctness of the design. All devices are real with their parasitic provided by the foundry, including MOS transistors, MIM capacitors, on-chip spiral inductors, and resistors. No idealized components are included, such as the current sources shown in above circuits.

Figure 5 shows the tuning characteristic of this circuit. The minimum oscillation frequency is $1.75 \mathrm{GHz}$, while the maximum oscillation frequency is $3.16 \mathrm{GHz}$, achieving a tuning range of $57 \%$, much wider than the works previously published.

The amplitude regulation function is also verified. The reference voltage is set to make the peak-to-peak value of the oscillation amplitude stabilized at about $0.6 \mathrm{~V}$. Figure 6 shows the stabilization process of the oscillation amplitude when the tuning voltage is set to zero. It can be seen that the initial amplitude reaches near $4 \mathrm{~V}$, which is close to the gate break-down voltage. After about $800 \mathrm{~ns}$, the amplitude is stabilized and regulated to about $0.6 \mathrm{~V}$.

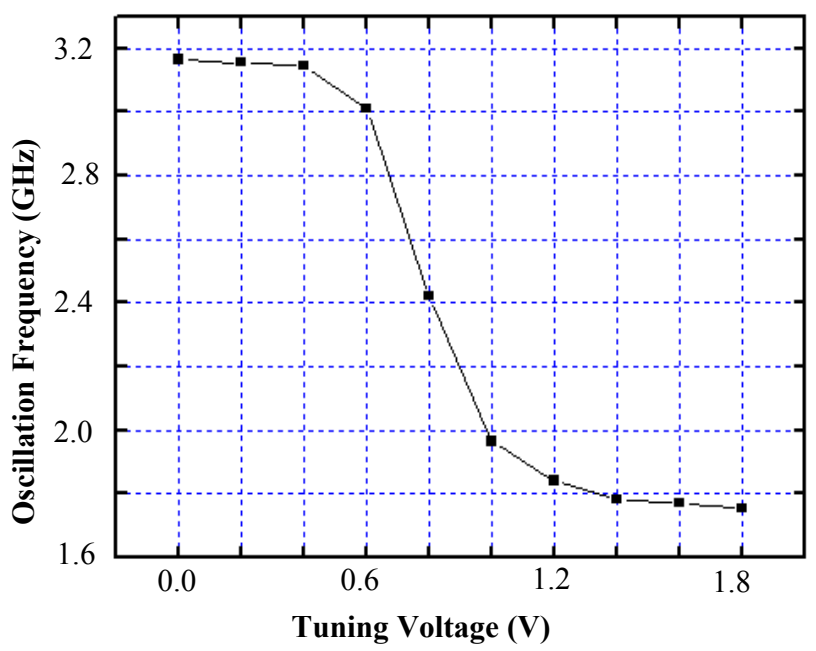

Figure 5. Simulated oscillator tuning characteristic

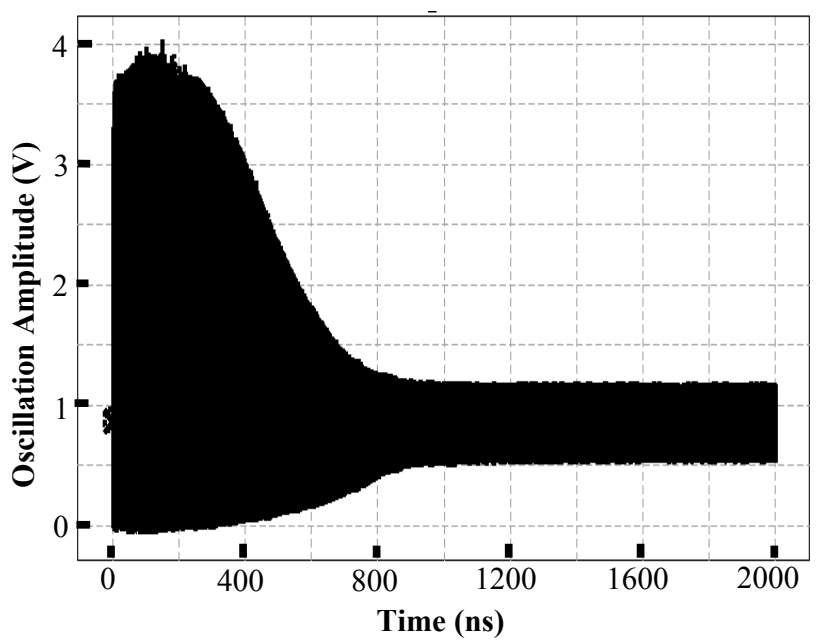

Figure 6. Stabilization process of the oscillation amplitude

The oscillation amplitude as a function of tuning voltage is also studied with the results shown in Figure 7. It can be seen that without automatic amplitude control, the amplitude variation exceeds $2.8 \mathrm{~V}$ over the whole tuning range, and the maximum amplitude reaches about $4 \mathrm{~V}$, exceeding twice of the power supply voltage. While with the automatic amplitude control, the amplitude variation is about $0.15 \mathrm{~V}$ over the whole tuning range, and the amplitude is stabilized to about $0.6 \mathrm{~V}$. 


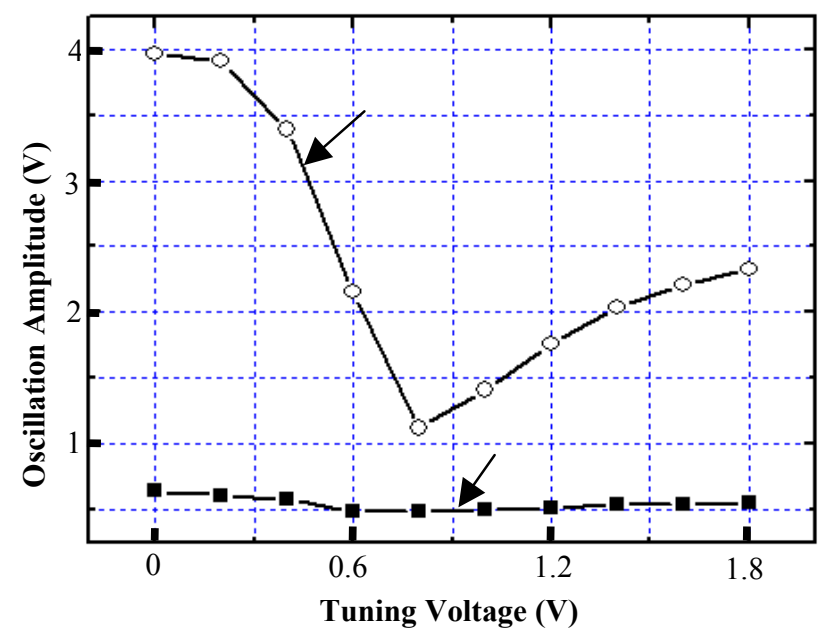

Figure 7. Comparasion of the oscillation amplitude without and with automatic amplitude control

\section{CONCLUSION}

This paper discusses the circuit design of a wide tuning range CMOS VCO with automatic amplitude control by using the mixed-signal CMOS technology. By tuning the resistance in series with a capacitor, a tuning range of over $50 \%$ has been achieved for a CMOS VCO. To ensure stable oscillation amplitude over the whole tuning range, a constant amplitude control loop is also incorporated in this circuit. The novel tuning principle is explained, and then the implementation details and design considerations of this circuit follows. After all, the simulation results are proposed with all real devices models provided by the foundry including their parasitic, showing the correctness of our design.

\section{REFERENCES}

[1] Thomas H. Lee, The Design of CMOS Radio-Frequency Integrated Circuits, Cambridge University Press, 1998, England, chapter 16.

[2] Nilakantan Seshan, Jay Rajagopalan, Kartikeya Mayaram, "Design of Low Power 2.4GHz CMOS LC Oscillators with Low Phase Noise and Large Tuning Range", in Proceedings of ISCAS'2002, Vol. IV, pp. 409-412, Phoenix, Arizona, U. S. A., May 2002.

[3] Fikret Dulger, Edgar Sanchez-Sinencio, "Design Trade-offs of a Symmetric Linearized CMOS LC VCO", in Proceedings of ISCAS'2002, Vol. IV, pp. 397-400, Phoenix, Arizona, U. S. A., May 2002.

[4] Jishnu Bhattacharjee, Debanjan Mukherjee, Joy Laskar, "A Monolithic CMOS VCO for Wireless LAN Applications", in Proceedings of ISCAS'2002, Vol. IV, pp. 441-444, Phoenix, Arizona, U. S. A., May 2002.

[5] Chan-Hong Park, Beomsup Kim, "A Low-Noise, 900MHz VCO in 0.6 um CMOS", IEEE Journal of Solid-State Circuits, vol. 34, No. 5, May 1999, pp. 586-591.

[6] Frank Herzel, Heide Erzgraber, Nikolay Ilkov, "A New Approach to Fully Integrated CMOS LC-Oscillators with a Very Large Tuning Range", in Proceedings of CICC'2000, pp.573-576.

[7] C. Samori, A. L. Lacaita, A. Zanchi, S. Levantino, G. Cali, "Phase Noise Degradation at High Oscillation Amplitudes in LC-Tuned VCO's", IEEE Journal of Solid-State Circuits, vol. 35, No. 1, January 2000, pp. 96-99.

[8] Dandan Li, Yannis P. Tsividis, "A Loss-Control Feedback Loop for VCO Indirect Tuning of RF Integrated Filters", vol. 47, No. 3, March 2000, pp. 169-175.

[9] Bernabe Linares-Barranco, Teresa Serrano-Gotarredona, “ A Loss Control Feedback Loop for VCO Stable Amplitude Tuning of RF Integrated Filters", in Proceedings of ISCAS'2002, Vol. I, pp. 521-524, Phoenix, Arizona, U. S. A., May 2002. 